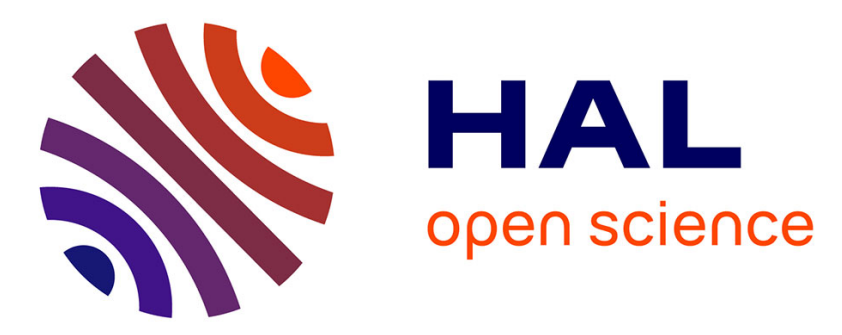

\title{
Regularised crack behaviour effects on continuum modelling of quasi-brittle materials under cyclic loading
}

Maxime Vassaux, Benjamin Richard, Frédéric Ragueneau, Alain Millard

\section{To cite this version:}

Maxime Vassaux, Benjamin Richard, Frédéric Ragueneau, Alain Millard. Regularised crack behaviour effects on continuum modelling of quasi-brittle materials under cyclic loading. Engineering Fracture Mechanics, 2015, 149, pp.19. 10.1016/j.engfracmech.2015.09.040 . hal-01271756

\section{HAL Id: hal-01271756 \\ https://inria.hal.science/hal-01271756}

Submitted on 10 Feb 2016

HAL is a multi-disciplinary open access archive for the deposit and dissemination of scientific research documents, whether they are published or not. The documents may come from teaching and research institutions in France or abroad, or from public or private research centers.
L'archive ouverte pluridisciplinaire HAL, est destinée au dépôt et à la diffusion de documents scientifiques de niveau recherche, publiés ou non, émanant des établissements d'enseignement et de recherche français ou étrangers, des laboratoires publics ou privés. 


\section{Regularised crack behaviour effects on continuum modelling of quasi-brittle materials under cyclic loading}

ARTICLE in ENGINEERING FRACTURE MECHANICS · SEPTEMBER 2015

Impact Factor: 1.77 · DOI: 10.1016/j.engfracmech.2015.09.040

READS

49

4 AUTHORS, INCLUDING:

Maxime Vassaux

Aix-Marseille Université

10 PUBLICATIONS 5 CITATIONS

SEE PROFILE

F. Ragueneau

Ecole normale supérieure de Cachan

89 PUBLICATIONS 543 CITATIONS

SEE PROFILE

\section{Benjamin Richard}

Atomic Energy and Alternative Energies Co...

51 PUBLICATIONS 147 CITATIONS

SEE PROFILE 


\title{
Regularised crack behaviour effects on continuum modelling of quasi-brittle materials under cyclic loading
}

\author{
M. Vassaux ${ }^{\mathrm{a}}$, B. Richard ${ }^{\mathrm{b}, *}$, F. Ragueneau ${ }^{\mathrm{a}}$, A. Millard $^{\mathrm{c}}$ \\ ${ }^{a}$ LMT-Cachan (ENS Cachan/CNRS/PRES UniverSud Paris) \\ 61, avenue du Président Wilson, 94230 Cachan, France \\ ${ }^{b} C E A, D E N$, DANS, DM2S, SEMT, Laboratoire d'Études de Mécanique Sismique \\ F-91191 Gif-sur-Yvette, France \\ ${ }^{c} C E A, D E N, D A N S$, DM2S, SEMT, Laboratoire de Mé Systèmes et Structures \\ F-91191 Gif-sur-Yvette, France
}

\begin{abstract}
A continuum model for quasi-brittle materials able to reproduce mechanical phenomena related to cyclic loading is proposed. Specific care is taken to reproduce progressive stiffness recovery and hysteresis effects caused respectively by crack closure and friction. A virtual testing approach is set up to analyse the evolution of microscopic quantities during uni-axial cyclic tests and to justify an original and efficient modelling of these phenomena. Thus, a regularised formulation of the homogenised multiple contact problem induced by the non-simultaneous closure of microscopic cracks is presented. The proposed continuum model is validated by means of member-scale simulations of reversely loaded reinforced concrete shear walls.
\end{abstract}

Keywords: damage; virtual testing; hysteresis effects; regularised stiffness recovery; concrete; cyclic loading;

\section{Introduction}

The design of robust and accurate constitutive models for quasi-brittle materials accounting for cyclic loading effects is an essential step in the process of predicting the response of civil engineering structures under complex loading, and more specifically earthquakes.

Several modelling techniques have been developed at the macroscopic scale to depict quasi-brittle materials behaviour.

i) Empirical models $[1,2,3,4]$ have been established following the publication of first uni-axial experimental results provided by [5]. Empirical models have therefore been developed mostly within a uni-axial framework. The empirical description of uni-axial cyclic behaviour of quasi-brittle

\footnotetext{
* Corresponding author

Email address: benjamin.richard@cea.fr (B. Richard)
} 
materials is highly representative of experimental behaviour, however the lack of derivability of the constitutive equations and their uni-axial formulation prevent them from being used to simulate full three-dimensional structures.

ii) Micro-mechanical models $[6,7]$, on the contrary, provide a sound physical framework. Constitutive equations obtained from homogenisation reproduce accurately experimentally observed phenomena. Despite such features, micro-mechanical models have hardly ever been employed for large scale structural simulations because they are highly computationally demanding implementation, in particular when homogenisation is realised numerically. Furthermore, micro-mechanical models including cyclic effects are still unavailable for three-dimensional simulations $[8,9]$.

iii) Phenomenological models $[10,11,12,13,14,15]$, at last, thanks to well developed theories such as continuum damage and plasticity, offer not only a sound physical framework which is consistent with thermodynamic principles, but also reasonable computational costs within a three-dimensional framework. However they still suffer from a lack of numerical robustness and of an accurate description of cyclic loading effects.

Toward the completion of three-dimensional concrete structures subjected to cylic loading simulations, phenomenological models have inherent advantages. However, the complexity of constitutive equations increases rapidly when trying to reproduce phenomena observed under uni-axial cyclic loading. Indeed even recently developed models are either not robust enough to simulate the complete response of structures subjected to cyclic loading, or do not reproduce accurately phenomena related to crack closure and friction (see fig. 1), as outlined by results of the ConCrack international benchmark [16].

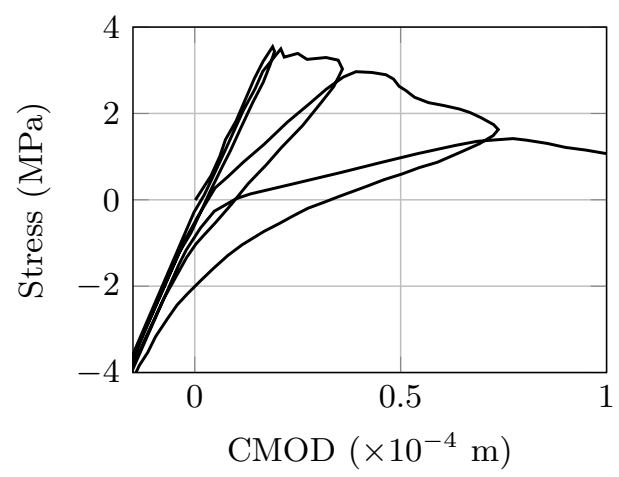

Figure 1: Experimental material's response under cyclic uni-axial loading [17].

Based on the uni-axial response of the phenomenological model proposed in [18] (see fig. 2), a number of issues of phenomenological models impairing structural computations related to cyclic loading can be highlighted:

- low regularity of the constitutive laws (discontinuous stiffness variations 
at stress sign changes) due to the consideration of stiffness recovery under cyclic loading, later leading to numerical robustness issues $\underline{19,20]}$;

- inaccurately reproduced hysteresis loops (associated stress-strain state, shape) when compared with experiments (see fig. 1), then implying poorly estimated hysteretic dissipation.

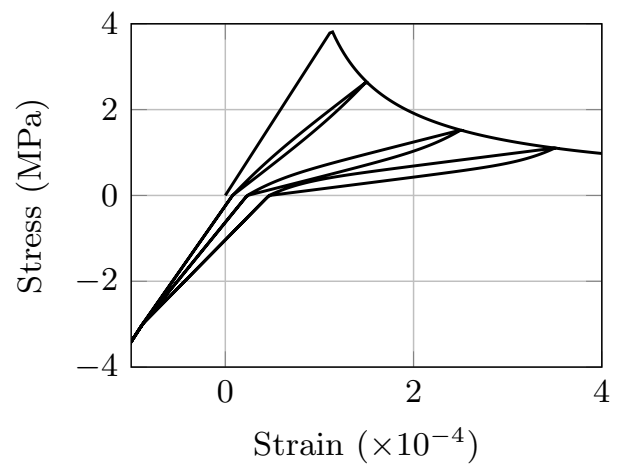

Figure 2: Simulated material response under cyclic uni-axial loading [18].

The first objective of this study is to enhance the efficiency of the model proposed in [18] to achieve structural simulations. Specific attention is paid to phenomena observed under cyclic loading. Issues encountered with the current model are partly induced by a lack of experimental data, preventing a finer mechanical description of the material behaviour from being established. Experimental data on quasi-brittle materials Representative Volume Element (RVE) subjected to cyclic loading are sparse due to control and repeatability issues. Therefore the replacement of some laboratory experimentation by virtual testing is investigated. A RVE is considered here to be approximately of a $0.1 \mathrm{~m}$ characteristic length, such as the material can be considered homogeneous with respect to its different phases (i.e five times the size of the biggest aggregate). The second objective of this paper is to illustrate the use of virtual testing to complement laboratory experimentation to develop regularised and accurate macroscopic constitutive models for quasi-brittle materials suited for cyclic loading. A microscopic model based on the Discrete Element Method (DEM) is utilised as a virtual testing machine [21]. Mesh dependencies of the computed results, using the macroscopic model and the Finite Element Method (FEM), will not be addressed in this paper. Techniques to circumvent such dependencies being already numerous in the literature [22, 23, 24, 25].

The paper is outlined as follows. First, the microscopic model is briefly introduced and a cyclic uni-axial test is performed. Second, the formulation of the macroscopic model is established. Third, the cyclic features, as well as mutli-axial fracture, of the macroscopic continuum model are characterised or calibrated by means of the virtual reference, that is the microscopic model. 
Fourth, local integration algorithm and local validation of the proposed continuum model are shown. Finally, the macroscopic continuum model is utilised in member-scale simulations of the reinforced concrete shear wall of the ConCrack international benchmark [26], which serve as validation.

\section{Virtual testing machine}

\subsection{Microscopic modelling of quasi-brittle materials}

For the virtual testing of samples at the material scale, a microscopic model has been used. The model is a $2 \mathrm{D}$ particle-based model made of a combination of a network of Euler-Bernoulli beams [27, 28, 29, 30, 31], which is used to reproduce cohesion and the fracture mechanisms between particles, and of the DEM [32, 33], which allows realistic description of crack interactions thanks to the integration of contact and friction mechanisms. Lattice and particles are generated respectively thanks to Delauney triangulation and Voronoi tessellation of the numerical sample (see fig. 3). Nodes generation being random, implying irregular lattice and particles shape, further simulations results are always obtained from averaging multiple mesh draws of the same test results (e.g 50). The microscopic model is developed within a quasi-static framework to enable reasonable computational costs and to avoid the introduction of arbitrary dynamic effects. The integration algorithm is an incremental version of classic event driven integration schemes, to allow the computation of the solution as a succession of stable equilibrium states, while accounting for other non-linearities than fracture, namely contact and friction, which cannot be solved in a event driven fashion.

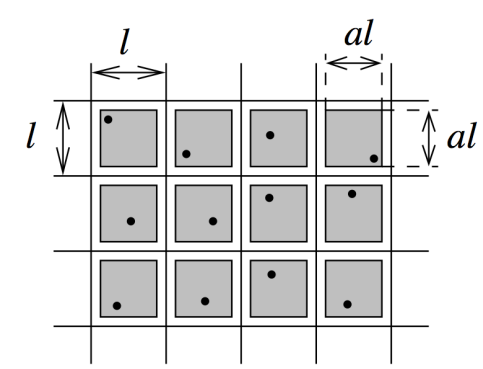

(a) random disposition of centroid nodes

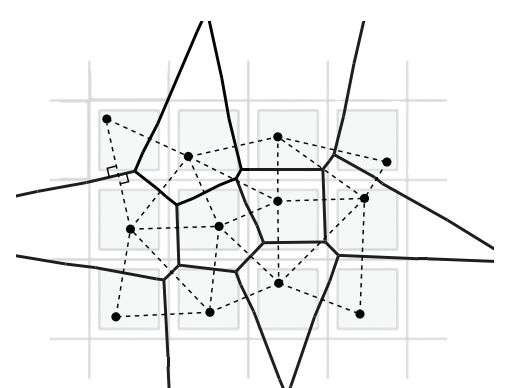

(b) bi-dimensional polygonal mesh generated by Voronoi tessellation from the left centroids distribution

Figure 3: Particles and lattice generation

This microscopic model as well as its integration algorithm are described in detail in [21]. The validation of the model under multi-axial and cyclic loading, and therefore with respect to mixed-mode fracture and contact mechanisms, has been presented in [34]. The calibration procedure, also presented in [35], has intentionally been kept simple so as to ease the use of the microscopic model 
as a virtual testing machine. The procedure can be achieved solely relying on an elastic compression test to calibrate the two elastic parameters $(\alpha, \bar{E})$ of the Euler-Bernoulli beams of the lattice (see eq. 1), a three-point bending test and a splitting test to calibrate the two scale factors $\left(\lambda_{\epsilon^{c r}}, \lambda_{\theta^{c r}}\right)$ and the common shape factor $(k)$ of the statistical distributions of the two thresholds $\left(\epsilon^{c r}, \theta^{c r}\right)$ utilised in beams failure criterion (see eq. 2), and a confined compression test to calibrate the friction coefficient $(\mu)$ of the Coulomb frictional sliding threshold (see eq. 3). Alternative calibration procedure for lattice model can be found in [36].

$$
\underline{F}_{c o h, i j}=\left\{\begin{array}{l}
F_{N, i j}=\frac{\bar{E} A_{b, i j}}{l_{b, i j}}\left(\underline{u}_{i}-\underline{u}_{j}\right) \cdot \underline{n}_{b, i j} \\
F_{T, i j}=\frac{12 \bar{E} I_{b, i j}}{l_{b, i j}^{3}}\left(\underline{u}_{i}-\underline{u}_{j}\right) \cdot \underline{t}_{b, i j}-\frac{6 \bar{E} I_{b, i j}}{l_{b, i j}^{2}}\left(\theta_{i}-\theta_{j}\right) \\
M_{Z, i j}=\frac{6 \bar{E} I_{b, i j}}{l_{b, i j}^{2}}\left(\underline{u}_{j}-\underline{u}_{i}\right) \cdot \underline{t}_{b, i j}+\frac{4 \bar{E} I_{b, i j}}{l_{b, i j}}\left(\theta_{i}-\frac{\theta_{j}}{2}\right)
\end{array}\right.
$$

where $F_{N, i j}, F_{T, i j}$ and $M_{Z, i j}$ stand for, respectively, the normal force, the tangent force and the bending moment in the beam linking particles $i$ and $j ; \underline{u}_{i}$ and $\theta_{i}$, respectively, the displacements and the rotation of the particle $i ; \underline{n}_{b, i j}$ and $\underline{t}_{b, i j}$, respectively, for the normal and tangent vectors to the cross-section of the beam linking particles $i$ and $j$; and $A_{b, i j}, I_{b, i j}$ and $l_{b, i j}$, respectively, the cross-section, the moment of inertia, and the length.

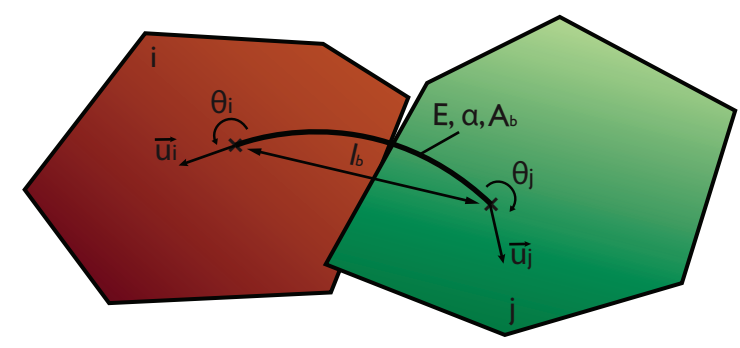

Figure 4: Two cohesively linked particles

$$
\frac{\epsilon_{i j}}{\epsilon_{i j}^{c r}}+\frac{\left|\theta_{i}-\theta_{j}\right|}{\theta_{i j}^{c r}}>1
$$

where $\epsilon_{i j}$ stands for the strain in the beam linking particles $i$ and $j$.

$$
\left\|\underline{F}_{f r i c, i j}\right\|=\min \left(\left\|\underline{F}_{f r i c, i j}^{e l}\right\|, \mu\left\|\underline{F}_{c o n t, i j}\right\|\right)
$$

where $\underline{F}_{c o n t, i j}$ and $\underline{F}_{f r i c, i j}$ stand respectively for the normal and the tangent frictional forces between two contacting particles $i$ and $j$. 
The entirety of the following simulations computed with the microscopic model calibrated solely using experimental results provided in [37], parameters value is recapped in the table 1.

\begin{tabular}{|c|c|c|c|c|c|c|}
$l_{p}(\mathrm{~m})$ & $\alpha$ & $\bar{E}(\mathrm{GPa})$ & $\lambda_{\epsilon^{c r}}$ & $\lambda_{\theta^{c r}}$ & $k$ & $\mu$ \\
\hline 0.002 & 0.83 & 46 & $4.3410^{-4}$ & $1.8510^{-3}$ & 1.8 & 0.7
\end{tabular}

Table 1: Calibrated value of the microscopic model's parameters for concrete based on standard tests from [37].

\subsection{Simulation of a uni-axial cyclic test}

The microscopic model is used to simulate a uni-axial cyclic test on concrete, from which the results will later serve as a reference and be exploited to characterise observed phenomena such as progressive stiffness recovery while unloading the specimen. Once again, it is important to note that results from such tests are sparse and only quantitative in the literature. Induced cyclic effects are strongly dependent on the test setup as well as on the concrete microscopic structure, and only few experimental results are available $[5,17]$, therefore there does not exist such thing as a reference experimental result for uni-axial cyclic tests.

The simulated setup is taken from the experiments reported in [17] (see fig. 5). Only the concrete specimen is modelled using the microscopic model, loading supports are not included in the simulation since their mechanical and geometrical characteristics are not known. The concrete specimen is a rectangular prism of $0.3 \mathrm{~m}$ height, and $0.15 \mathrm{x} 0.15 \mathrm{~m}^{2}$ cross-section. The specimen presents two notches at mid-height along the depth. 


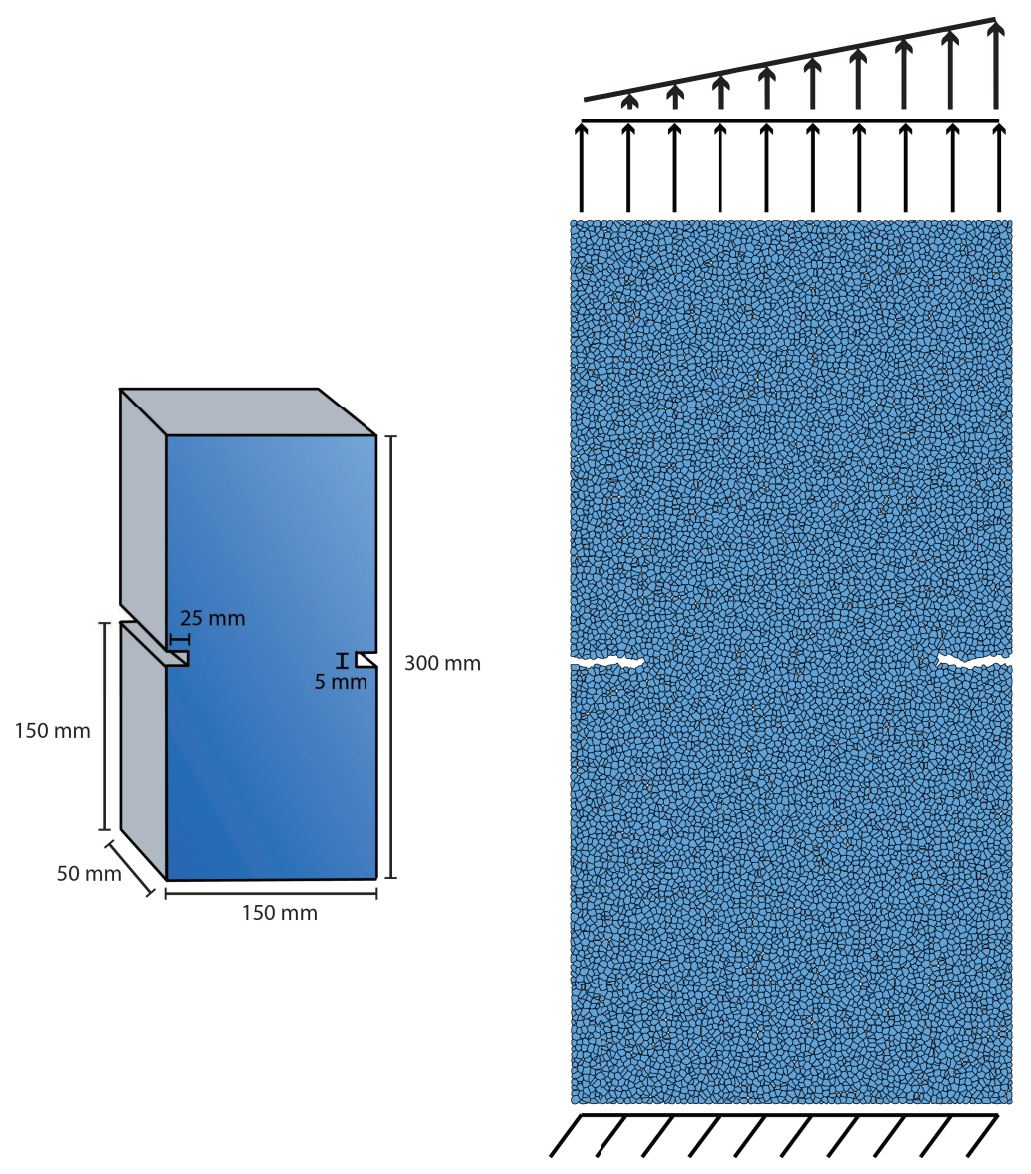

Figure 5: Uni-axial cyclic test experimental and numerical setups [17].

Boundary conditions are prescribed directly at the specimen's top and bottom ends following prescribed and measured motions during experimental testing in [17]. Prescribed displacement consists in successive cycles of damaging tension and elastic compression (see fig. 6). Prescribed rotation is extrapolated from experimental measurements of the rotation at each cycle's tensile peak load (see fig. 7) and is applied as linear gradient of displacement on the top of the sample (see fig. 5).

In between measured values, the rotation is considered to evolve linearly with respect to pseudo-time. 


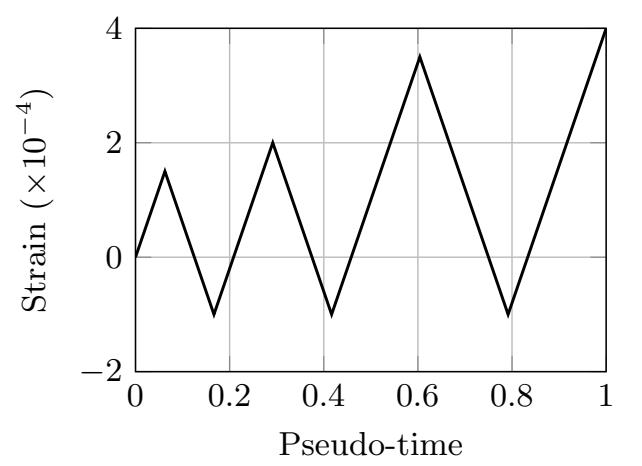

Figure 6: Applied axial strain during the uni-axial cyclic test [17].

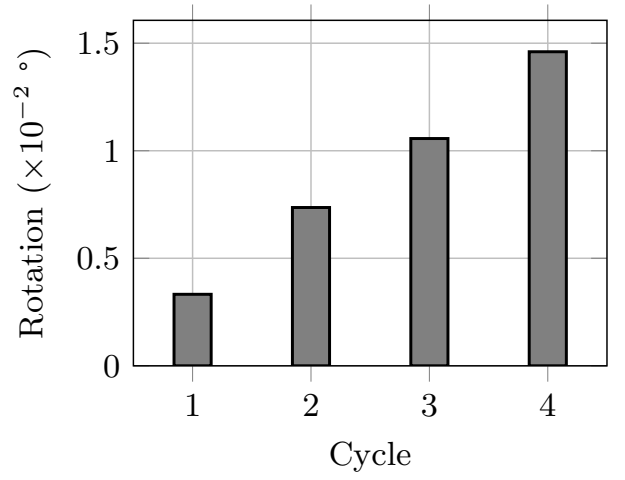

Figure 7: Measured rotation of the top surface of the sample after each loading cycle during the uni-axial cyclic test [17].

The macroscopic stress-strain response obtained through this simulation is presented in figure 8 . The response features qualitatively all the phenomena usually observed during a uni-axial cyclic test : progressive stiffness recovery at the tension to compression transition; crescent-shaped hysteresis loops; and residual strains alleviated in compression. The microscopic model result can be compared qualitatively to experimental results (see fig. 1), although only crack mouth opening displacement (sample notches average spacing) is provided. The crack pattern obtained at the end of this simulation is also provided (see fig. 9). 


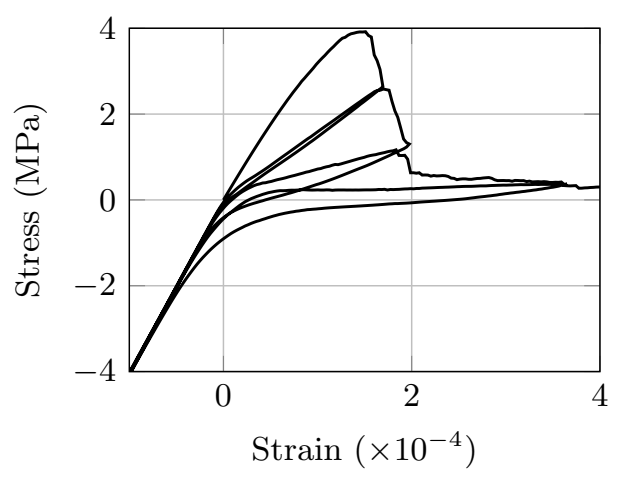

Figure 8: Simulated material's response under cyclic uni-axial loading with the microscopic model.

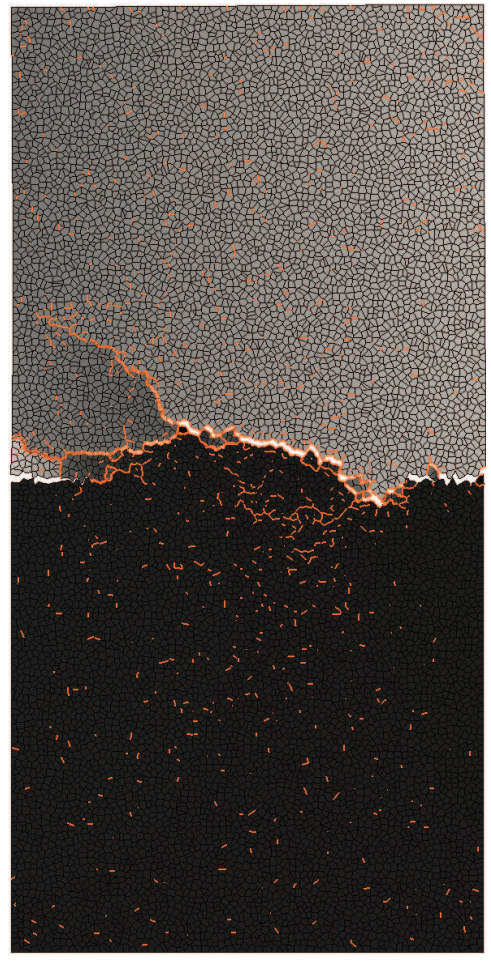

Figure 9: Uni-axial cyclic test crack pattern at the end of the simulation.

Boundary conditions have an important influence on tension test response [31]. It should be noted that if the rotation of the specimen's ends had been unrestrained, observed phenomena would have not been so intense. Rotation of the specimen's ends is necessarily associated to these phenomena, however it can either be a cause (misalignment of the actuators), or a consequence (asymmetric 
crack closure along the specimen section due to, for example, early age effects [38]). Nevertheless, such microscopic mechanisms are not clearly understood, which justifies the choice to directly impose rotations at specimen's ends.

The reference simulation setup is now established. The general formulation of the macroscopic model is now proposed. Results obtained from the reference simulations will later be used to characterise the progressive stiffness recovery phenomena.

\section{Formulation of the continuum constitutive model}

The phenomenological macroscopic model is formulated using a rather classic decomposition of the total stress in the RVE, as in [15]. It is considered that the total stress $\underline{\underline{\sigma}}$ can be split in two independent parts:

$$
\underline{\underline{\sigma}}=\underline{\underline{\sigma}}^{m}+\underline{\underline{\sigma}}^{f}
$$

with the stress in the cracked continuum medium $\underline{\underline{\sigma}}^{m}$, neglecting any interaction between cracks, classically modelled with a damage model; and the stress in cracks when closed $\underline{\sigma}^{f}$.

Helmholtz's free energies $\Psi^{m}$ and $\Psi^{f}$ respectively associated with the two stress tensors are defined, and compose the total free energy of the specimen:

$$
\Psi=\Psi^{m}+\Psi^{f}
$$

At this point, both thermodynamic potentials remain completely unspecified. The free energy $\Psi^{m}$ is completely detailed in the next paragraph based on existing models. In contrast, the free energy $\Psi^{f}$ is partially specified, the virtual testing method described in the next section is required to establish its formulation entirely.

\subsection{Modelling of fracture processes}

Fracture processes are modelled by means of the continuum damage theory. In general, the simpler the damage variable is kept, the more robust the proposed macroscopic model is. Therefore, in view of the structural applications of the proposed model, an isotropic damage model is formulated, implying a unique scalar damage variable. The free energy associated to the cracked continuum medium simply writes:

$$
\Psi^{m}=\frac{1}{2}(1-D) \underline{\underline{\epsilon}}: \boldsymbol{C}: \underline{\underline{\epsilon}}+\Psi^{m, D}(z)
$$

with $D$ the isotropic damage variable, $\underline{\underline{\epsilon}}$ the second-order total strain tensor, $\boldsymbol{C}$ the fourth-order Hooke's tensor, $z$ the isotropic hardening variable, and $\Psi^{m, D}$ the energy locked through the damage process, also called consolidation function. 
The formulation of the non-associated pseudo-potential of dissipation $\varphi^{m}$ is based a Mazars-like failure criterion [39] expressed in terms of energy variables.

$$
\varphi^{m}=\bar{Y}-\left(Y_{0}+Z\right)
$$

where $Z$ stands for the thermodynamic force associated to $z, \bar{Y}$ the energy rate $\bar{Y}=\frac{1}{2} E \epsilon_{0} \epsilon^{e q}$ which is written as a function of Mazars equivalent strain $\epsilon^{e q}=\sqrt{\langle\underline{\underline{\epsilon}}\rangle_{+}:\langle\underline{\underline{\epsilon}}\rangle_{+}}, \epsilon_{0}$ the elastic limit strain, and $Y_{0}$ the elastic limit energy rate written in a similar fashion $Y_{0}=\frac{1}{2} E \epsilon_{0}^{2}$.

The asymmetry between traction and compression loading is only considered through its consequence on the peak load and the softening behaviour of the material, and is introduced in the damage variable evolution law derived from $\Psi^{m, D}$. The formulation of $\Psi^{m, D}$ is adapted from the formulation proposed in [40]. An additional variable $\kappa$ is introduced to consider the effect of a confining pressure on crack propagation, namely a higher peak load value and a more ductile behaviour:

$$
\frac{\mathrm{d} \Psi^{m, D}}{\mathrm{~d} z}(z)=-\frac{\kappa}{B_{0}} \ln \left[\frac{Y_{0}}{\bar{Y}}(1+z)\right]
$$

where $B_{0}$ stands for a parameter controlling the softening behaviour and $\kappa$ computed as follows:

$$
\kappa=1+k_{0}\left(\frac{\left\langle\underline{\underline{\sigma}}^{m}\right\rangle_{-}:\left\langle\underline{\underline{\sigma}}^{m}\right\rangle_{-}}{\left(\underline{\underline{\sigma}}^{m}\right):\left(\underline{\underline{\sigma}}^{m}\right)}\right)^{1 / 2}=1+k_{0}\left(\frac{\langle\boldsymbol{C}: \underline{\underline{\epsilon}}\rangle_{-}:\langle\boldsymbol{C}: \underline{\underline{\epsilon}}\rangle_{-}}{(\boldsymbol{C}: \underline{\underline{\epsilon}}):(\boldsymbol{C}: \underline{\underline{\epsilon}})}\right)^{1 / 2}
$$

where $k_{0}$ stands for a parameter measuring the influence of the confining pressure, and therefore only influences the failure behaviour when cracks are induced indirectly (e.g. in compression). The direct tension behaviour remains uninfluenced, indeed $\kappa$ is then equals to 1 .

Based on consistency conditions $\left(\varphi^{m}, \dot{\varphi}^{m}\right)$ the damage variable evolution law may finally be written:

$$
D=1-\frac{Y_{0}}{\bar{Y}} \exp \left[-\frac{B_{0}}{\kappa}\left(\bar{Y}-Y_{0}\right)\right]
$$

\subsection{Modelling of crack closure phenomena}

Constitutive laws related to cyclic effects are formulated in two distinct steps. First, a non-linear elastic modelling is utilised to reproduce the progressive crack closure and the regain of stiffness induced by initiated contacts in cracks when unloading a damaged sample in tension. Second, a dissipative mechanism is introduced by means of plastic model utilised to reproduce friction initiated at the surface of closing cracks and associated hysteresis effects.

\subsubsection{Stiffness recovery}

The mechanical behaviour of cracks described by the stress tensor $\underline{\underline{\sigma}}^{f}$ is first considered elastic. $\underline{\sigma}^{f}$ is defined as a non-linear function of the strain tensor 
$\underline{\underline{\epsilon}}^{f}=D \times \underline{\underline{\epsilon}}$, which could be called the homogenised contribution of crack openings $\overline{\bar{t}}$ the total strain of the RVE. Such strain tensor definition may also be found in [41].

The following assumption is made on the evolution of $\underline{\underline{\sigma}}^{f}$ with respect to $\underline{\underline{\epsilon}}^{f}$ :

$$
\underline{\underline{\dot{\sigma}}}^{f}=\vartheta\left(\underline{\underline{\epsilon}}^{f}\right) \boldsymbol{C}: \underline{\underline{\epsilon}}^{f}
$$

The function $\vartheta$ is chosen to be a scalar, in other words, the tangent modulus of the cracks stress-strain relationship is proportional to the undamaged Hooke's elastic tensor. Therefore $\vartheta$ represents the part of the lost stiffness due to cracking which is recovered thanks to crack closure, and can only take values ranging from 0 (when cracks are completely opened) to 1 (when cracks are completely closed). Since $\vartheta$ evolves according to the materials loading state, it is considered to be dependent on $\underline{\underline{\epsilon}}^{f}$.

Set aside its physical meaning, $\vartheta$ can be considered as a numerical regularisation of the multiple Signorini's contact problem [42] induced by crack closure. $\vartheta$ should then be formulated to evolve from 0 to 1 in sufficiently regular manner to avoid discontinuities of the constitutive laws or of their derivatives. The final formulation of $\vartheta$ is proposed and physically justified in the next section by means of a virtual analysis of the evolution of the proportion of closed cracks during a uni-axial cyclic test.

As a function of $\underline{\epsilon}^{f}$, the scalar aspect of $\vartheta$ is obtained by means of a scalar indicator of this strain tensor. A simple indicator dependent on the sign of the loading is required, so as to observe stiffness recovery passing from tension to compression. Therefore, the first invariant is used. The elastic part of the free energy associated to crack behaviour is then written as:

$$
\Psi^{f, e}=\int_{t=0}^{t=T}\left(\int_{t=0}^{t=T} \vartheta\left(\mathrm{I}_{1}\left(\underline{\underline{\epsilon}}^{f}\right)\right) \boldsymbol{C}: \frac{\mathrm{d} \underline{\underline{\epsilon}}^{f}}{\mathrm{~d} t} \mathrm{~d} t\right) \frac{\mathrm{d} \underline{\underline{\epsilon}}^{f}}{\mathrm{~d} t} \mathrm{~d} t
$$

An initial condition can be set for $\vartheta$, in order to ensure that the free energy $\Psi^{f, e}$ is $\mathcal{C}^{2}$, such condition would be that $\vartheta$ remains integrable $\left(\mathcal{C}^{0}\right)$.

\subsubsection{Hysteresis effects}

The explanation of hysteresis effects relying on frictional sliding occurring at the cracks surfaces justifies a modelling method based on plasticity theory [15]. In consequence, a perfect plasticity model along with a Drucker-Prager criterion is utilised (see eq. 13).

$$
\varphi^{f}=\sqrt{\mathrm{J}_{2}\left(\underline{\underline{\sigma}}^{f}\right)}+\mu_{0} \mathrm{I}_{1}\left(\underline{\underline{\sigma}}^{f}\right)
$$

where $\mu_{0}$ stands for a parameter which could be assimilated to a "friction coefficient".

Because of perfect plasticity, the free energy $\Psi^{f}$ is reduced to an elastic part $\Psi^{f, e}$ and introduces a single internal variable, the plastic strain accumulated 
through sliding between cracks $\underline{\underline{\epsilon}}^{f, p}$, defined as $\underline{\underline{\epsilon}}^{f}=\underline{\underline{\epsilon}}^{f, e}+\underline{\underline{\epsilon}}^{f, p}$ :

$$
\begin{aligned}
\Psi^{f} & =\Psi^{f, e}\left(\underline{\underline{\underline{\epsilon}}}^{f}-\underline{\underline{\epsilon}}^{f, p}\right) \\
& =\int_{t=0}^{t=T}\left(\int_{t=0}^{t=T} \vartheta\left(\mathrm{I}_{1}\left(\underline{\underline{\underline{\epsilon}}}^{f}-\underline{\underline{\epsilon}}^{f, p}\right)\right) C: \frac{\mathrm{d}\left(\underline{\underline{\epsilon}}^{f}-\underline{\underline{\epsilon}} f, p\right)}{\mathrm{d} t} \mathrm{~d} t\right) \frac{\mathrm{d}\left(\underline{\underline{\epsilon}}^{f}-\underline{\underline{\epsilon}}^{f, p}\right)}{\mathrm{d} t} \mathrm{~d} t
\end{aligned}
$$

A non-associated dissipation potential is utilised to compute flow directions:

$$
\bar{\varphi}^{f}=\sqrt{\mathrm{J}_{2}\left(\underline{\underline{\sigma}}^{f}\right)}
$$

Thus, the plastic strain $\underline{\epsilon}^{f, p}$ refers to an isochoric transformation and its first invariant is null. In addition, $\underline{\underline{\epsilon}}^{f, p}$ is independent on $\underline{\underline{\epsilon}}^{f}$. Then, $\vartheta\left(\mathrm{I}_{1}\left(\underline{\underline{\epsilon}}^{f}-\underline{\underline{\epsilon}}^{f, p}\right)\right)=$ $\vartheta\left(\mathrm{I}_{1}\left(\underline{\underline{\epsilon}}^{f}\right)\right)$, hence:

$$
\Psi^{f}=\int_{t=0}^{t=T}\left(\int_{t=0}^{t=T} \vartheta\left(\mathrm{I}_{1}\left(\underline{\underline{\underline{f}}}^{f}\right)\right) \boldsymbol{C}: \frac{\mathrm{d} \underline{\underline{\epsilon}}^{f}}{\mathrm{~d} t} \mathrm{~d} t\right) \frac{\mathrm{d} \underline{\underline{\epsilon}}^{f}}{\mathrm{~d} t} \mathrm{~d} t-\frac{1}{2} \vartheta\left(\mathrm{I}_{1}\left(\underline{\underline{\epsilon}}^{f}\right)\right) \underline{\underline{\epsilon}}^{f, p}: \boldsymbol{C}: \underline{\underline{\epsilon}}^{f, p}
$$

Finally:

$$
\Psi^{f}=\Psi^{f, e}\left(\underline{\underline{\epsilon}}^{f}\right)-\frac{1}{2} \vartheta\left(\mathrm{I}_{1}\left(\underline{\underline{\epsilon}}^{f}\right)\right) \underline{\underline{\epsilon}}^{f, p}: C: \underline{\underline{\epsilon}}^{f, p}
$$

Conditions to ensure that the free energy $\Psi^{f}$ is $\mathcal{C}^{2}$ are not changed by the introduction of frictional sliding, it is still required that $\vartheta$ be $\mathcal{C}^{0}$.

Regarding the physical significance of the chosen criterion, the $\mathrm{J}_{2}$ part refers directly to shear occurring in cracks, while $\mathrm{I}_{1}$ rather refers to cracks surface normal pressure. In consequence, when $\mathrm{J}_{2}$ exceeds $\mu_{0} \mathrm{I}_{1}$, frictional sliding is observed. Furthermore, the $\mathrm{J}_{2}$ part depends on $\vartheta$ through $\underline{\underline{\sigma}}^{f}$, and therefore depends on the proportion of closed cracks. Thus when all the cracks are opened the $J_{2}$ is negligible and as expected no friction force is observed.

\section{Virtual testing applications}

\subsection{Characterisation of crack closure}

The formulation of the function $\vartheta$ will now be defined. This process is undertaken using the virtual testing machine aforementioned and the discrete simulations of the uni-axial cyclic test.

The function $\vartheta$, as the proportion of closed cracks, represents the proportion of cracks in which forces are transmitted across the crack faces and contribute to the stiffness recovery of the specimen. The evolution of $\vartheta$ is characterised by analysing the evolution of the ratio of number of contacts detected and the number of cracks initiated in the virtual material sample during the simulation 
of the uni-axial cyclic test. The analysis is carried out for different damage levels, that is different maximum crack strains $\epsilon_{\max }^{f}$. The maximum crack strain tensor is defined as $\underline{\epsilon}_{\max }^{f}=\underline{\epsilon}^{f, t_{m}}$, where $t_{m}$ stands for the pseudo-time such as $\mathrm{I}_{1}\left(\underline{\underline{\epsilon}}^{f, t_{m}}\right)=\max _{t_{0} \leq \bar{t} \leq t}\left[\mathrm{I}_{1}\left(\underline{\epsilon}^{f, \bar{t}}\right)\right]$, where $t$ stands for the current pseudo-time. The figure 10 shows the evolution of the proportion of closed cracks during unloading phases.

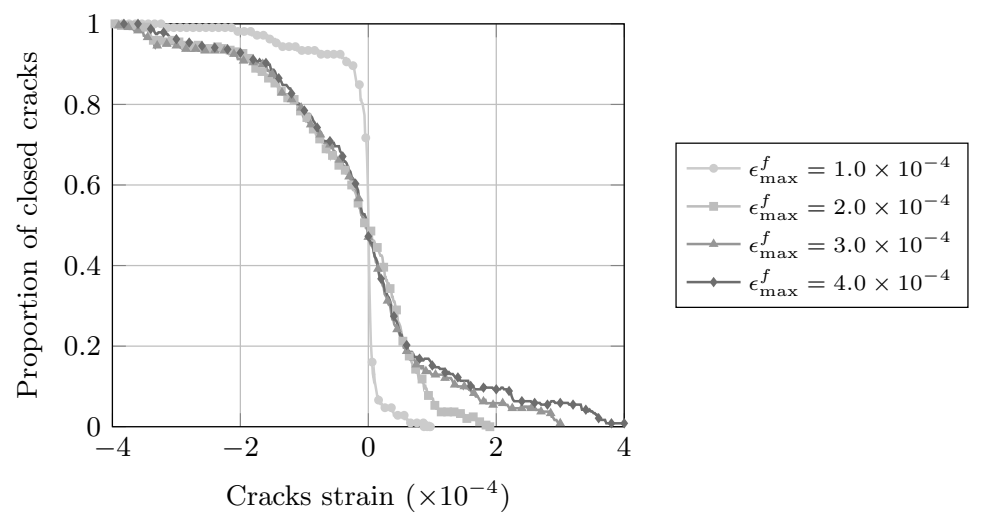

Figure 10: Dependency of the proportion of closed cracks to $\epsilon_{\max }^{f}$ evaluated with the microscopic model during unloading.

Independently of $\epsilon_{\max }^{f}$, the proportion of closed cracks follows a sigmoidal evolution with respect to $\epsilon^{f}$. To determine an analytical expression for $\vartheta$ let us consider the probabilistic event "a crack closes", $\vartheta$ is the distribution function of this event. From the results obtained with the microscopic model, it appears that this event follows a symmetrical distribution centred on $\epsilon^{f}=0$, therefore it could be assumed that the event "a crack closes" follows a Gaussian distribution of zero mean. $\vartheta$ is then expressed as a Gaussian distribution function:

$$
\vartheta=1-\frac{1}{1+\exp \left[-f \times \mathrm{I}_{1}\left(\underline{\underline{\epsilon}}^{f}\right)\right]}
$$

where the function $f$ is associated with the variance of the Gaussian distribution.

The maximum crack strain $\epsilon_{\max }^{f}$ affects the evolution of the proportion of closed cracks (see fig. 10). The more damaged the specimen, the bigger the variance of the event "a crack closes". The function $f$ is finally chosen to account for this dependency:

$$
\vartheta=1-\frac{1}{1+\exp \left[-\frac{\alpha_{0}}{\mathrm{I}_{1}\left(\underline{\underline{\epsilon}}_{\max }^{f}\right)} \mathrm{I}_{1}\left(\underline{\underline{\epsilon}}^{f}\right)\right]}
$$

where $\alpha_{0}$ stands for a parameter controlling a reference variance of the event "a cracks closes". 


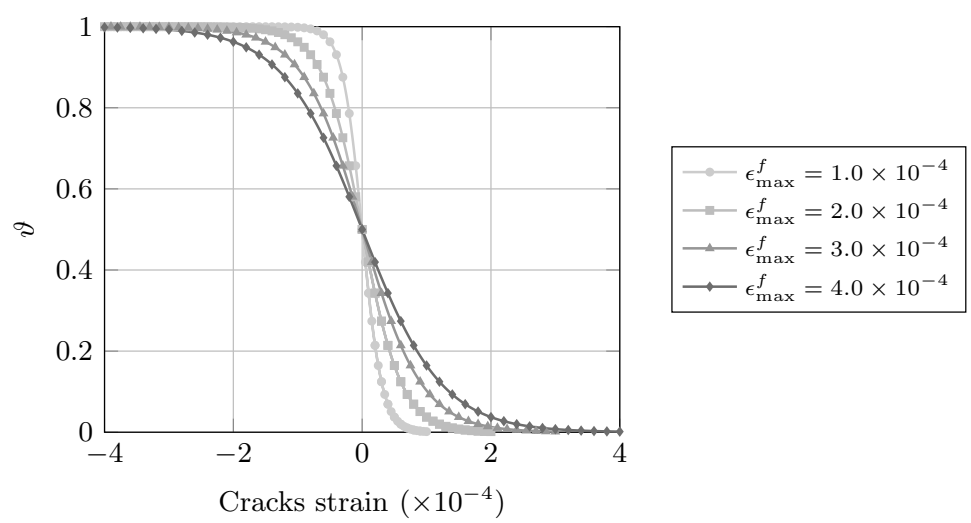

Figure 11: Dependency of the proportion of closed cracks to $\epsilon_{\max }^{f}$ evaluated with the function $\vartheta$ for $\alpha_{0}=6.5$ during unloading.

The formulated expression of $\vartheta$ is a sigmoidal function, therefore is continuously integrable. Continuity of the total free energy is therefore ensured.

Now that the expression of $\vartheta$ is defined, the expression of the cracks stress rate tensor (11) can be integrated, leading to the following expression of the cracks stress tensor:

$$
\underline{\underline{\sigma}}^{f}=C:\left[\underline{\underline{\epsilon}}^{f}-\frac{\underline{\epsilon}_{\max }^{f}}{\alpha_{0}} \times \log \left(1+\exp \left[-\frac{\alpha_{0}}{\mathrm{I}_{1}\left(\underline{\underline{\epsilon}}_{\max }^{f}\right)} \mathrm{I}_{1}\left(\underline{\underline{\epsilon}}^{f}\right)\right]\right)\right]
$$

\subsection{Calibration of the frictional sliding}

Unlike crack closure, efficient and robust modelling techniques already exist to reproduce frictional sliding at the macroscopic scale based on plasticity theory. Although, the introduced parameters remain to be calibrated. As the Drucker-Prager criterion for the initiation of sliding between cracks has already been presented (see eq. 13), the parameter $\mu_{0}$ has to be evaluated. Instead of calibrating the value of $\mu_{0}$ arbitrarily, a methodology based on the virtual testing machine is once again proposed.

By means of the microscopic model it is possible to estimate the dissipated energy specific to frictional sliding, among other dissipation mechanisms. Therefore, the parameter $\mu_{0}$ is calibrated in order to make the friction specific dissipation in concrete RVEs modelled using the microscopic model and the macroscopic continuum model (i.e a Gauss point) identical.

The comparison of both models is realised during the simulation of a complete cycle of uni-axial cyclic test, namely loading, unloading, reloading. The amplitude of the cycle is arbitrarily chosen to vary from $\epsilon_{\max }^{f}=2.0 \times 10^{-4}$ to $\epsilon_{\max }^{f}=-1.0 \times 10^{-4}$ back to $\epsilon_{\max }^{f}=2.0 \times 10^{-4}$. Such amplitudes correspond to strains where the most dissipation through friction, and therefore hysteresis effects, is expected due to cracks opening and closure. 
Friction related dissipated energies are respectively computed from the following formulations:

- for the microscopic discrete model, the dissipated energy is incrementally computed as the sum of the variations of dissipated energies for every contact between particles $i$ and $j$ detected, thus:

$$
\begin{aligned}
E_{\mu}^{d, t+1}=E_{\mu}^{d, t}+\sum_{i=1, \ldots, n_{\text {particules }}} & \\
& {\left[\sum_{j=1, \ldots, n_{\text {contact }}^{i}} \frac{1}{2}\left(T\left(\underline{F}_{f r i c, i j}^{t+1}+\underline{F}_{f r i c, i j}^{t}\right) \cdot\left(\Delta \delta u_{s, i j} \underline{t}_{c, i j}\right)^{t+1}\right)\right] }
\end{aligned}
$$

where $\underline{F}_{f r i c, i j}$ and $\Delta \delta u_{s, i j}$ respectively stand for the friction force and the increment of relative sliding displacement between particles $i$ and $j, t$ the current time-step, and $n_{\text {contact }}^{i}$ the amount of contacts detected on the particle $i$;

- for the macroscopic continuum model, the dissipated energy is incrementally computed as the integral over the representative volume $\Omega$ of the tensor product of the cracks stress $\underline{\underline{\sigma}}^{f}$ and the increment of plastic cracks strain $\Delta \underline{\underline{\epsilon}}^{f}$, thus:

$$
E_{\mu}^{c, t+1}=E_{\mu}^{c, t}+\frac{1}{2} \int_{\Omega}\left[\left(\underline{\underline{\sigma}}^{f, t+1}+\underline{\underline{\sigma}}^{f, t}\right): \Delta \underline{\underline{\epsilon}}^{f, p, t+1}\right] d V
$$

Evolutions of the computed energies during the aforementioned loading cycle are presented in figure 12. Both dissipated energies remain null during the loading step, cracks are completely opened. Then, a significant increase is observed during unloading, while cracks progressively close. During reloading, both energies evolutions stagnate first since cracks are completely closed, before opening progressively leading to another increase of the dissipated energy related to friction. 


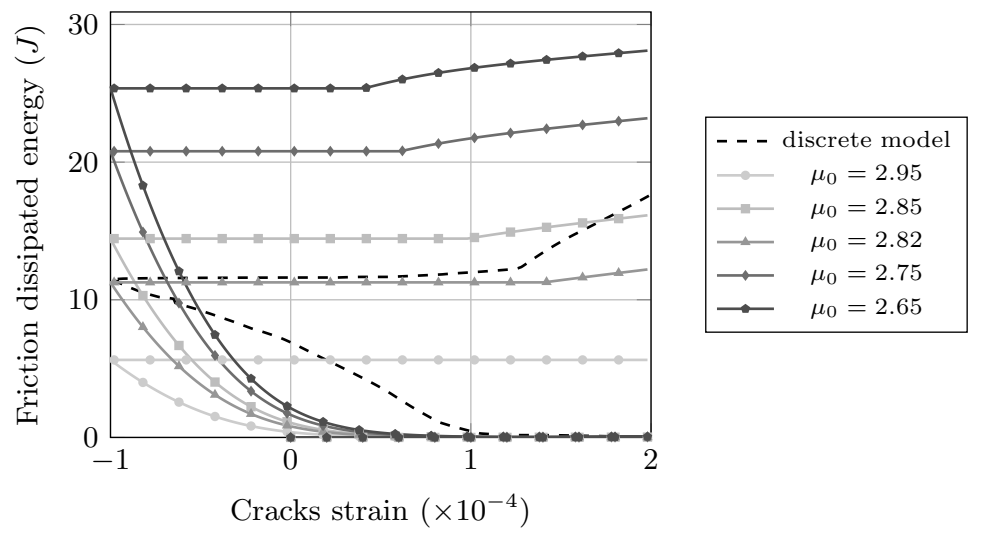

Figure 12: Sensitivity of the friction related dissipated energy of the continuum model to the parameter $\mu_{0}$.

The parameter $\mu_{0}$ is then evaluated at $\mu_{0}=2.82$. It is added that the friction related dissipated energies computed with both models present similar trends, which provides confidence in the choice of the perfect plastic modelling, along with a Drucker-Prager criterion, of crack frictional sliding mechanism.

\subsection{Calibration of multi-axial failure}

The parameter $k_{0}$ presented in the formulation of the continuum medium behaviour affects directly multi-axial failure behaviour as explained above. However, the parameter $k_{0}$ is intricate to calibrate since it is used to adjust energy dissipation depending on the loading case, unlike $Y_{0}$ or $B_{0}$, respectively analytically linked to the limit elastic limit strain and the fracture energy. Therefore, $k_{0}$ is calibrated so as to fit the complete response (peak-load and softening behaviour) of loading path involving several fracture modes (see fig. 14).

Member-scale simulations described below involve the simulation of a shear wall. Therefore, to ensure a quantitative shear response of the macroscopic model, the calibration of $k_{0}$ is carried out on material-scale shear test. The virtual testing machine is then taken advantage of to produce a reference result (see fig. 13 and 14), which similarly to uni-axial cyclic tests remain rare in the literature. 


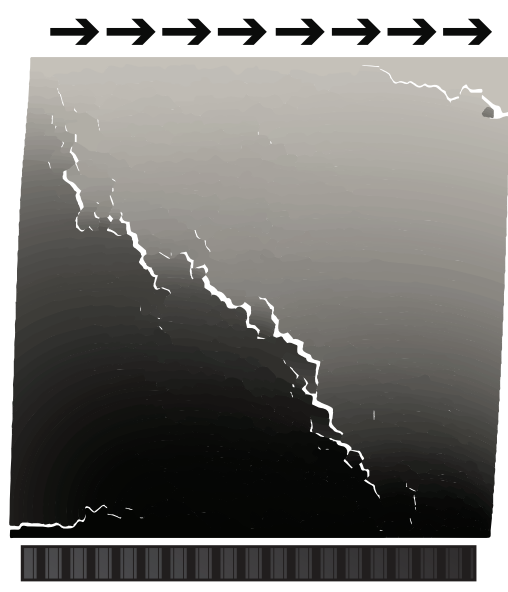

Figure 13: Simple shear test simulation with the virtual testing machine.

The parameter $k_{0}$ is then evaluated at $k_{0}=4.5$. It has to be noted that when $k_{0}$ equals unity the damage model used to describe the continuum medium is exactly equivalent to Mazars model [39]. The introduction of the function $\kappa$ and therefore of the parameter $k_{0}$, are thus justified by allowing the multi-axial failure behaviour to be controlled, and specifically shear failure behaviour. This behaviour is rarely accurately reproduced with a unique scalar damage variable.

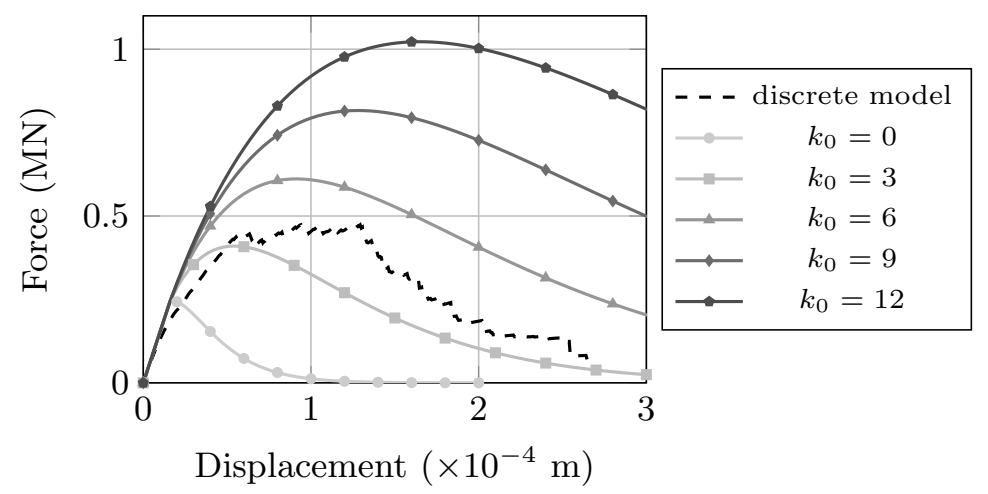

Figure 14: Sensitivity of the simple shear response of the continuum model to the parameter $k_{0}$.

\section{Local integration algorithm}

The integration algorithm of the complete model, including damage, nonlinear elasticity and plasticity is presented in figure 15. The algorithm is explicit with respect to damage and non-linear elasticity, and implicit with respect to plasticity. 


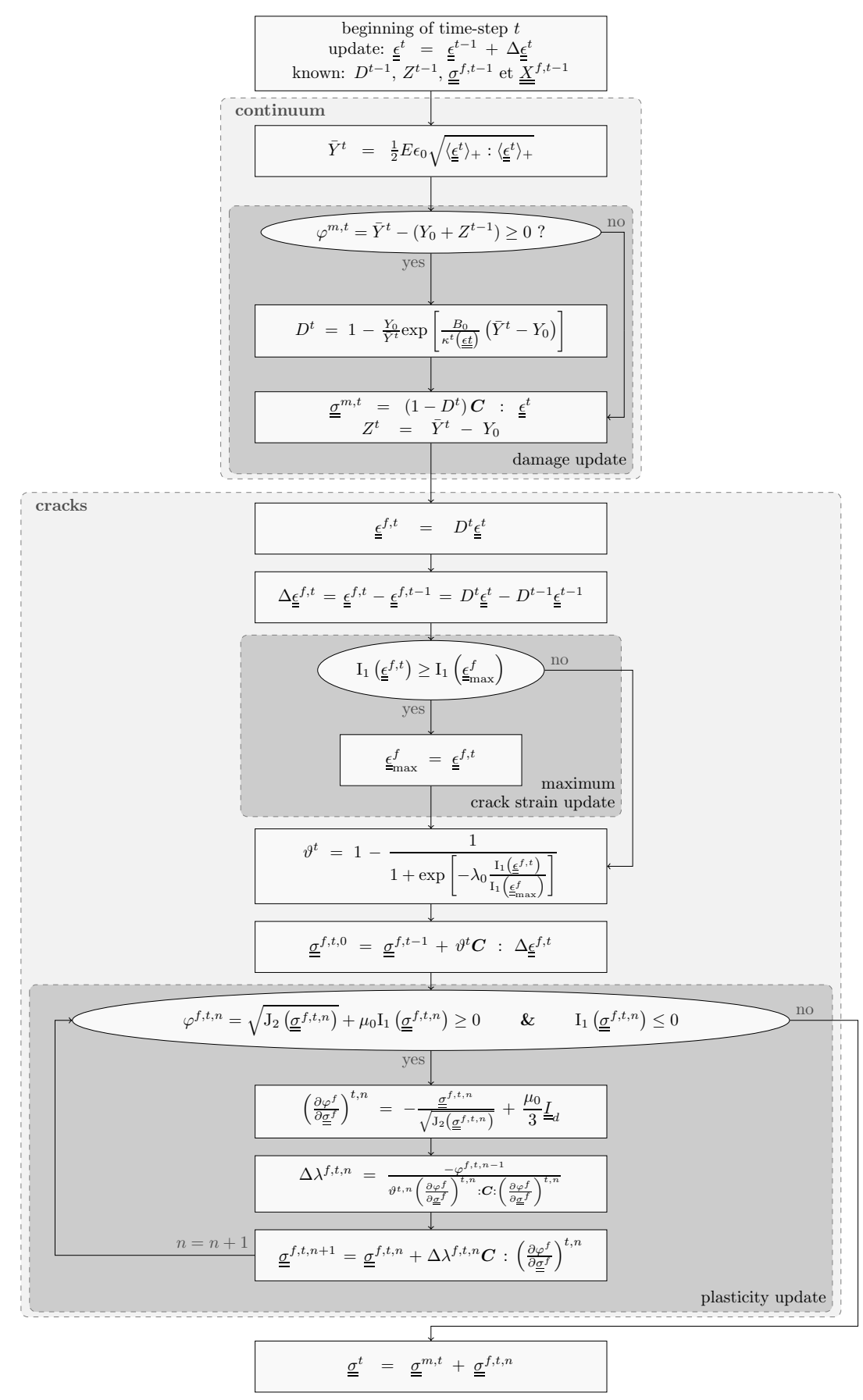

Figure 15: Flowchart of the integration algorithm of the complete continuum model. 


\section{Local responses of the continuum model}

Parameters of the continuum medium part of the model are calibrated by equivalence with macroscopic reference results provided by the virtual testing machine on a square sample of $0.1 \mathrm{~m}$ side length. The tension resistance, the tension fracture energy, and the compression resistance are respectively utilised to calibrate $\epsilon_{0}, B_{0}$ and $k_{0}$ (see tab. 2). Throughout this section, results plotted with dashed lines refer to the results provided by the virtual testing machine, and plain lines refer to results obtained with the continuum model.

\begin{tabular}{|c|c|c|c|c|c|}
$E(\mathrm{GPa})$ & $\epsilon_{0}$ & $B_{0}\left(\mathrm{~kJ}^{-1} \cdot \mathrm{m}^{3}\right)$ & $k_{0}$ & $\alpha_{0}$ & $\mu_{0}$ \\
\hline 37 & $1.0 \times 10^{-4}$ & $4\left(G_{f}=56 \mathrm{~J} . \mathrm{m}^{-2}\right)$ & 4.5 & 6.5 & 2.82
\end{tabular}

Table 2: Calibrated values of the continuum model's parameters for the local validation.

\subsection{Uni-axial cyclic response}

The uni-axial cyclic test response obtained with the macroscopic model is fairly close to the reference response obtained with the virtual testing machine (see fig. 8 and 16). First, the addition of the non-linear crack-closure model allows to reproduce the progressive stiffness recovery as well as residual strains which disappear in compression (see fig. 16b). Second, the addition of the plastic model of crack behaviour, enables the emergence of the hysteretic behaviour, crescent-shaped hysteresis loops are observed at accurate stress levels (see fig. 16c). Thus, dissipative mechanisms are activated for appropriate solicitations amplitudes.
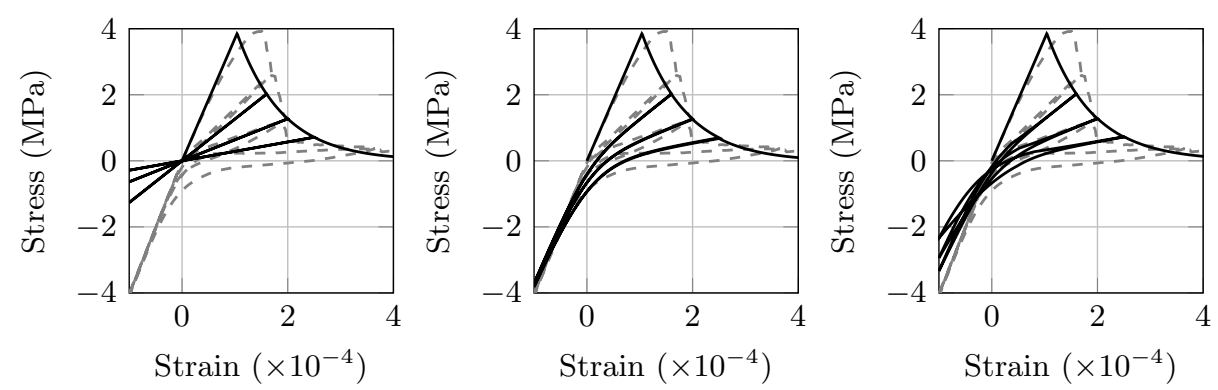

(a) without crack closure model(b) with elastic crack closure(c) with elasto-plastic crack clomodel sure model

Figure 16: Uni-axial cyclic response of the continuum model.

\subsection{Induced responses}

A uni-axial cyclic pure compression test is simulated (see fig. 17). Friction between loading supports and the sample strongly influences the inelastic compression response. Therefore, so as to ease the comparison, for both models, no friction in between the sample and the loading supports has been considered. 


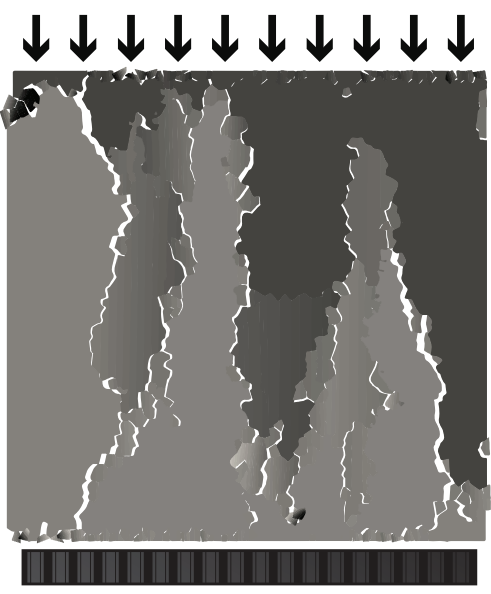

Figure 17: Pure compression test simulation with the virtual testing machine.

The overall compression response, peak-value and softening behaviour, obtained with the continuum model is similar to the response obtained with the virtual testing machine. Such results are interesting, knowing that a single scalar damage variable is introduced in the continuum model. It is also quite interesting to note the contribution of the crack behaviour to the compression response. Important hysteresis loops can be observed as a consequence of crack frictional sliding. Furthermore, crack behaviour increases the value of the compression resistance, due to the formulation of the function $\vartheta$, which depends on the first invariant of the cracks strain tensor.
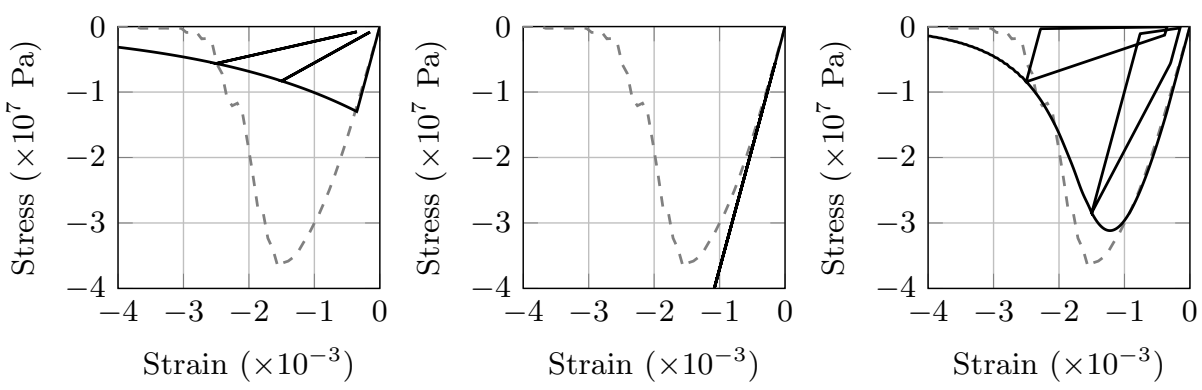

(a) without crack closure model(b) with elastic crack closure(c) with elasto-plastic crack clomodel sure model

Figure 18: Uni-axial compression cyclic response of the continuum model.

The failure enveloppe obtained with the continuum model is similar to the failure envelope of the discrete model (see fig. 19), as long as bi-compression loading paths are not considered. Once again due to the dependence of $\vartheta$ on the first invariant of the cracks strain tensor, even though damage increases, cracks 
are immediately considered as closed since the first invariant is highly negative. Thus, with this model, failure can never be reached for bi-compression loading paths if crack behaviour is accounted for.

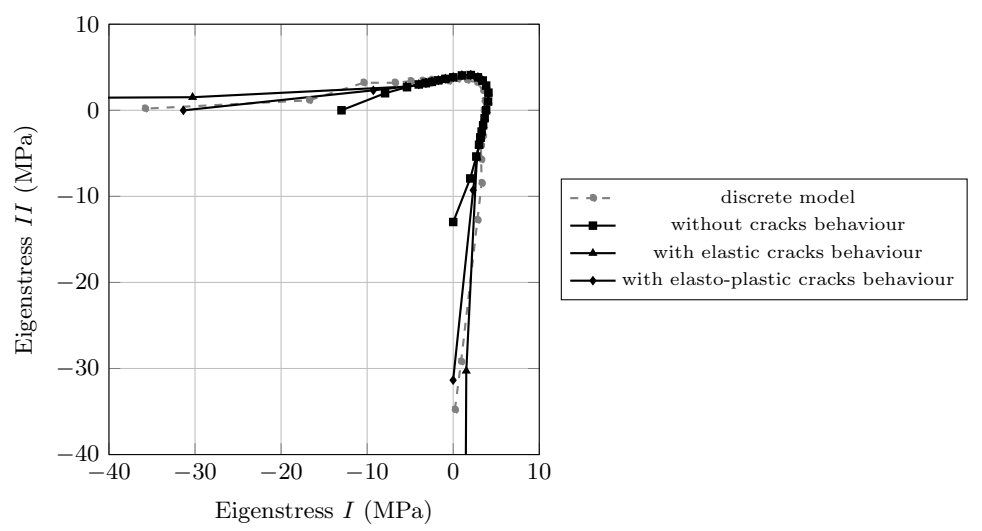

Figure 19: Failure enveloppe of the continuum model.

The simulation of uni-axial and bi-axial compression tests has shown that under negative hydrostatic pressures $\left(\mathrm{I}_{1}\left(\underline{\epsilon}^{f}\right) \leq 0\right)$, although the damage variable increases, its effects on stiffness and softening are immediately compensated by a value of $\vartheta$ close to 1 . Moreover, stiffness degradation can only be observed if sliding in cracks is accounted for and occurs, in other words if elasto-plastic behaviour of crack is considered and if the cracks stress is highly deviatoric. Indeed, stiffness degradation can be observed under uni-axial compression but never under bi-axial compression. When crack sliding occurs, since perfect plasticity is considered here, the shear stiffness associated to crack behaviour is lost. A consequence of this observation is that the "friction" coefficient $\mu_{0}$ highly influences the compression peak-load, independently of the tension behaviour.

\section{Structural validation of the continuum model}

The model is now validated using data from a reinforced concrete wall submitted to horizontal shear forces. The continuum model has shown interesting responses under tension, compression and shear, as well as cyclic loading, which is why the simulation of such structure is attempted. This structure has been tested in the ConCrack benchmark as part of the CEOS.fr project [43]. The simulation of this case study allows the testing of two aspects of the continuum model:

- its numerical robustness, because the setup implies local shear loading, multiple cracking locations, and structural size dimensions; in addition few accurate numerical results are available according to conclusions drawn from the ConCrack benchmark [16]; 
- its representativeness with respect to phenomena observed under cyclic loading, because cyclic loading is applied.

The proposed continuum model is utilised to describe the inelastic part of the wall, it is calibrated solely, with the exception of the few parameters given in the experimental report, using the virtual testing machine. Responses to standard tests are prescribed with the microscopic model and used as references for the calibration of the macroscopic continuum model's parameters.

The continuum model has been implemented within the three-dimensional finite element software Cast3M [44] developed at the French Sustainable Energies and Atomic Energy Commission (CEA) to simulate the complete structure's response.

\subsection{Modelling}

Details about the shear wall's geometry and a few materials properties can be found in $[43,26]$. Two mesh densities have been investigated, with elements side length $h^{c}=0.1 \mathrm{~m}$ or $h^{c}=0.03 \mathrm{~m}$.

The concrete structure is modelled using eight-noded hexahderal finite elements. The upper and lower parts of the wall, with increased thickness, are considered to remain elastic (in green, see fig. 21). The central thinner part of the wall is modelled with the proposed model (in gray, see 21). Inelastic parameters are entirely calibrated from results provided by the virtual testing machine, with the exception of the elastic strain limit, along with elastic parameters, which were given in the benchmark report [43]. Calibrated parameters value are given in the table 3. Fracture energy regularisation depending on elements size is implemented [22].

\begin{tabular}{|c|c|c|c|c|c|}
$E(\mathrm{GPa})$ & $\epsilon_{0}$ & $B_{0}\left(\mathrm{~kJ}^{-1} \cdot \mathrm{m}^{3}\right)$ & $k_{0}$ & $\alpha_{0}$ & $\mu_{0}$ \\
\hline 22 & $1.5 \times 10^{-4}$ & 4 & 4.5 & 6.5 & 2.82
\end{tabular}

Table 3: Calibrated values of the continuum model's parameters for the member-scale simulations.

The rebars are modelled using uni-axial Timoshenko beam elements in order to reproduce accurately the shear stiffness of the structure (in orange, red and purple, see fig. 21). Steel rebars are modelled using an isotropic plastic model, which parameters are directly taken from [43], that is, a 180 GPa Young's modulus, a $550 \mathrm{MPa}$ elastic stess limit and a $10 \%$ failure strain. Steel-concrete interface is assumed to be perfect. 


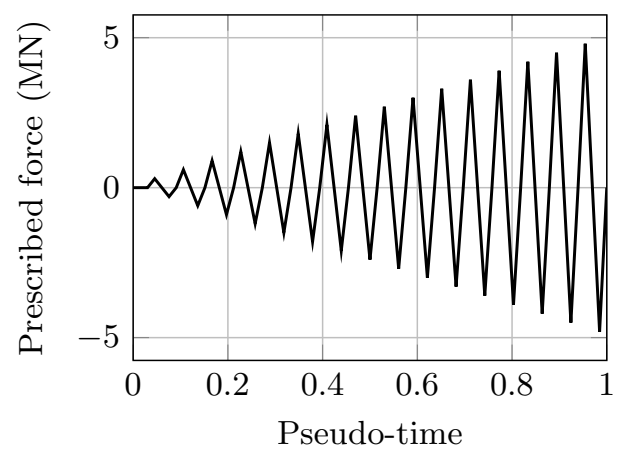

Figure 20: Prescribed load during the alternative cyclic test.

Horizontal forces are alternatively applied (see fig. 20) on the loading surfaces (in deep blue, see fig. 21) so as to only generate compression at the neighbourhood of the loading surfaces in the concrete.

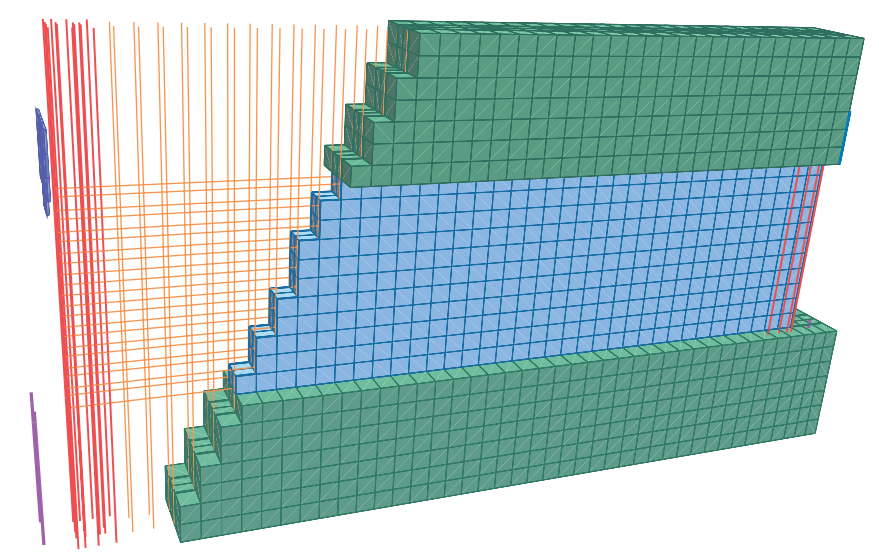

Figure 21: Mesh of the shear wall (with $h^{c}=0.1 \mathrm{~m}$ ).

\subsection{Simulation}

The global response obtained with the proposed model is satisfactory with respect to the two following aspects. The complete response to the prescribed load has been successfully simulated (see fig. 22b), while few complete threedimensional results have previously been obtained during the ConCrack benchmark [16]. This confirms the inherent numerical robustness of the continuum model. The achieved robustness is mostly explained by the continuity of the established constitutive laws, to which can be added to a fully explicitly local integrated continuum model with the exception of plasticity (see fig. 15).

Good representativeness is also noticed. Regarding the fracture mechanisms, simulated results show that the global response is well estimated, both the elastic limit or the stiffness of the cracked structure, are accurately evaluated. Regarding the cyclic effects, the global response is symmetrical, indeed stiffness 
recovery is accurately observed when the horizontal loading direction is inverted and peak loads are identical on both loading directions. This observation implies that the crack closure mechanism is accurately accounted for and reproduced even for member-scale simulations. Because of the isotropic damage description, if stiffness recovery had not been accurately reproduced, a symmetrical global response could not have been obtained. This consists in a first validation of the chosen $\vartheta$ formulation. The global response presents hysteresis loops, yet the steel-concrete interface is non-degradable and steel rebars remained elastic during the whole simulation. Consequently, the observed hysteresis loops are induced by the continuum model and confirms that frictional sliding is reproduced in member-scale simulations.

However some comments can be added regarding the representation of frictional sliding, which differs from experimented one (see fig. 22a). Therefore, this simulation is only a partial validation of the modelling of hysteresis effects. Nevertheless, such shape of hysteresis loops might be explained by mechanisms unrelated to concrete's behaviour, namely steel-concrete interface degradation and friction $[45,46,47]$.

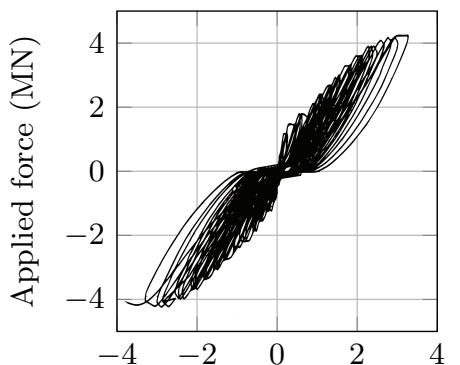

Horizontal displacement $(\mathrm{mm})$ (a) experiment

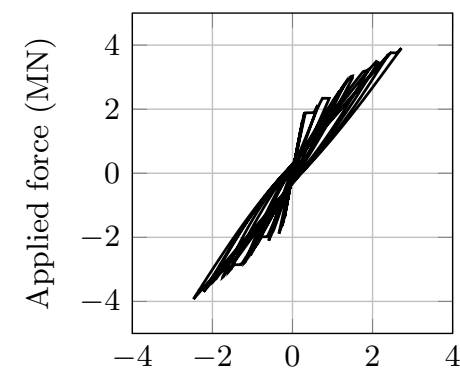

Horizontal displacement (mm)

(b) simulation

Figure 22: Global response of the shear wall to reverse loading.

Regarding fracture mechanisms, cracking patterns, although presenting accurate orientation of cracks, underestimate cracks density. Regarding cyclic effects, cracking patterns bring further validation of the mechanical description proposed in the continuum model. In addition to the symmetrical global response, symmetrical cracking patterns have been obtained even for high-loading cycles (see fig. 23). Furthermore, it can be noticed that when loading is inverted that longitudinal strain localisation associated to crack opening homogenises anew. In other words, cracked compressed zones of the wall behave as continuum due to crack closure and induced contact. These observations made on the cracking patterns are further validations of the accurate reproduction of crack closure mechanism. 

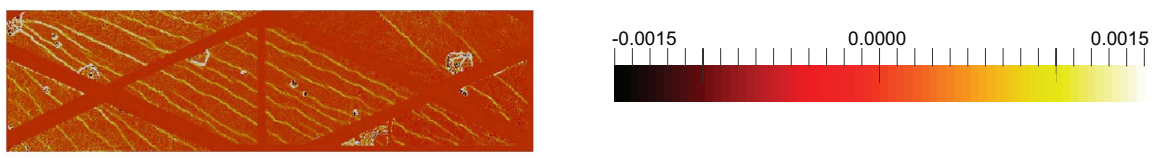

(a) experiment $\left(4.2 \mathrm{MN}, \epsilon_{x x} \times 5.10^{-2}\right)$

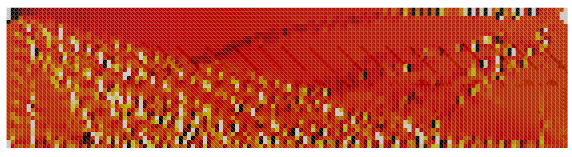

(c) simulation $\left(4.2 \mathrm{MN}, h^{c}=0.03 \mathrm{~m}\right)$

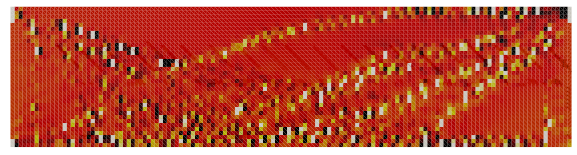

(d) simulation $\left(-4.2 \mathrm{MN}, h^{c}=0.03 \mathrm{~m}\right)$

Figure 23: Longitudinal strain field $\epsilon_{x x}$ in the shear wall under reverse loading.

\section{Conclusion}

The objective of the present paper was to propose a continuum model for quasi-brittle materials able to reproduce phenomena observed under cyclic loading, while remaining sufficiently robust to simulate the behaviour of real structures. The formulation of macroscopic constitutive laws has been established using a virtual testing approach. The virtual testing approach, based on a particular model, has been illustrated and proved to be efficient to characterise and calibrate quantities, which could not have been reached using laboratory experimentation: the proportion of closed cracks and the specific dissipated energy related to friction and even the response to a simple shear test.

A model framework for simulating quasi-brittle materials submitted to cyclic loading has been developed on the basis of the model proposed in [18]. The continuity of the original model has been improved introducing the distribution function of a statistical Gaussian process to regularise the homogenised contact problem induced by crack closure. However thermodynamical consistency of the model has not been proved. An homogenised cracks stress tensor, on which a perfect plastic model has been applied, to reproduce phenomena related to frictional sliding of cracks surfaces. Such modelling choices led to satisfactory results, namely crescent-shaped and accurately positioned hysteresis loops. The quality of the formulated and calibrated model has been validated at the member scale by the simulation shear wall under reverse loading. The simulation of the complete loading path has been achieved proving the numerical robustness of the proposed continuum model. The accurate consideration of crack closure and friction has also been observed at the member scale which served as a validation of the cyclic effects modelling.

A last comment should be added on the dissipative behaviour of the simulated structure. It has been explained that the continuum concrete model, is the main, if not the only, dissipation source in the structure model, since rebars have remained elastic during the whole simulation. Therefore, concrete's crack sliding is the only active dissipative mechanism during the simulation 
under quasi-static reverse cyclic loading. Such mechanical behaviour is typical of highly reinforced structures found in nuclear power plants. Even though hysteresis loops area is well underestimated when compared with experimental results, this area is clearly non-negligible. Efforts made towards the accurate reproduction of the dissipative mechanisms observed uni-axial cyclic loading are thus justified, even when member-scale simulations are considered.

\section{Acknowledgments}

The Nuclear Energy Division of the French Sustainable Energies and Atomic Energy Commission (CEA/DEN) and the École Normale Supérieure of Cachan (ENS-Cachan) are kindly thanked for financial supports. The research reported in this paper has been supported in part by the SEISM Paris Saclay Research

Institute and by the ILMAB project (Grant FUI11). Ludovic Jason is also thanked for providing the mesh of the simulated reinforced concrete wall.

\section{References}

[1] K. Gylltoft, A fracture mechanics model for fatigue in concrete, Matériaux et Construction 17 (1) (1984) 55-58.

[2] H. W. Reinhardt, H. A. W. Cornelissen, D. A. Hordijk, Tensile tests and failure analysis of concrete, Journal of Structural Engineering 112 (11) (1986) 2462-2477.

[3] D. Z. Yankelevsky, H. W. Reinhardt, Uniaxial behavior of concrete in cyclic tension, Journal of Structural Engineering 115 (1) (1989) 166-182.

[4] D. A. Hordijk, Local approach to fatigue of concrete, Ph.D. thesis, T.U. Delft, The Netherlands (1991).

[5] H. W. Reinhardt, H. A. W. Cornelissen, Post-peak cyclic behaviour of concrete in uniaxial tensile and alternating tensile and compressive loading, Cement and concrete research 14 (2) (1984) 263-270.

[6] R. A. Guyer, K. R. McCall, G. N. Boitnott, Hysteresis, discrete memory, and nonlinear wave propagation in rock: A new paradigm, Physical review letters 74 (17) (1995) 3491.

[7] V. Pensée, D. Kondo, L. Dormieux, Micromechanical analysis of anisotropic damage in brittle materials, Journal of Engineering Mechanics 128 (8) (2002) 889-897.

[8] S. Mertens, J. Vantomme, J. Carmeliet, Modelling of the influence of the damage on the behavior of concrete during tensile-compressive loading, Fracture mechanics of concrete structures.

[9] S. Mertens, Hysteresis, damage and moisture effects in quasi-brittle porous materials, Ph.D. thesis, K.U. Leuven, Belgium (2009). 
[10] P. Ladevèze, On an anisotropic damage theory, in: Proc. CNRS Int. Coll. 351 Villars-de-Lans, Failure criteria of structured media, J. P. Boehler ed. 1993, 1983, pp. 355-363.

[11] J. C. Simo, J. Ju, Strain and stress based continuum damage models formulation, International Journal of Solids and Structures 23 (1987) 821-840.

[12] G. Meschke, R. Lackner, H. A. Mang, An anisotropic elastoplastic-damage model for plain concrete, International Journal for Numerical Methods in Engineering 42 (4) (1998) 703-727.

[13] F. Ragueneau, C. La Borderie, J. Mazars, Damage model for concrete like materials coupling cracking and friction, contribution towards structural damping: first uniaxial application, Mechanics Cohesive Frictional Materials 5 (2000) 607-625.

[14] M. Matallah, C. La Borderie, Inelasticity-damage-based model for numerical modeling of concrete cracking, Engineering Fracture Mechanics 76 (8) (2009) 1087-1108.

[15] A. Sellier, G. Casaux-Ginestet, L. Buffo-Lacarrière, X. Bourbon, Orthotropic damage coupled with localized crack reclosure processing. part i: Constitutive laws, Engineering Fracture Mechanics 97 (2013) 148-167.

[16] L. Buffo-Lacarriere, C. Rospars, A. Delaplace, A. Duong, L. Jason, International benchmark concrack: Synthesis of the results, in: Proceeding of ConCrack 2 Workshop, ACI, 2011.

[17] O. Nouailletas, C. L. Borderie, C. Perlot, P. Rivard, G. Ballivy, Experimental study of crack closure on heterogeneous quasi-brittle materials, Journal of Engineering Mechanics (2015) 04015041.

[18] B. Richard, F. Ragueneau, Continuum damage mechanics based model for quasi brittle materials subjected to cyclic loadings: Formulation, numerical implementation and applications, Engineering Fracture Mechanics 98 (2013) 383-406.

[19] I. Mihai, A. Jefferson, Smoothed contact in a micromechanical model for cement bound materials, Computers \& Structures 118 (2013) 115-125.

[20] A. Jefferson, I. Mihai, The simulation of crack opening-closing and aggregate interlock behaviour in finite element concrete models, International Journal for Numerical Methods in Engineering.

[21] M. Vassaux, B. Richard, F. Ragueneau, A. Millard, A. Delaplace, Lattice models applied to cyclic behavior description of quasi-brittle materials: advantages of implicit integration, International Journal for Numerical and Analytical Methods in Geomechanics 39 (7) (2015) 775-798. 
[22] A. Hillerborg, M. Modeer, P. E. Petersson, Analysis of crack formation and crack growth in concrete by means of fracture mechanics and finite elements, Cement and Concrete Research 6 (1976) 773-782.

[23] G. Pijaudier-Cabot, Z. P. Bažant, Nonlocal damage theory, Journal of Engineering Mechanics 1113 (1987) 1512-1533.

[24] R. De Borst, H.-B. Mühlhaus, Gradient-dependent plasticity : formulation and algorithmic aspects, Internation Journal For Numerical Methods In Engineering 35 (1992) 521-539.

[25] C. Giry, F. Dufour, J. Mazars, Stress based nonlocal damage model, International Journal of Solids and Structures 48 (25-26) (2011) 3431-3443.

[26] C. Rospars, D. Chauvel, Ceos.fr experimental programme and reference specimen tests results, European Journal of Environmental and Civil Engineering 18 (7) (2014) 738-753.

[27] A. Hrennikoff, Solution of problems of elasticity by the framework method, Journal of applied mechanics 8 (4) (1941) 169-175.

[28] H. J. Herrmann, A. Hansen, S. Roux, Fracture of disordered, elastic lattices in two dimensions, Physical Review B 39 (1) (1989) 637-648.

[29] Z. P. Bažant, M. Tabbara, M. Kazemi, G. Pijaudier-Cabot, Random particle model for fracture of aggregate and fibre composites, Journal of Engineering Mechanics 116 (8) (1990) 1686-1705.

[30] E. Schlangen, J. G. M. Van Mier, Experimental and numerical analysis of micromechanisms of fracture of cement-based composites, Cement and Concrete Composites 14 (1992) 105-118.

[31] J. G. M. Van Mier, Fracture processes of concrete, Vol. 12, CRC press, 1996.

[32] P. A. Cundall, O. D. L. Strack, A discrete numerical model for granular assemblies, Geotechnique 29 (1979) 47-60.

[33] K. Meguro, M. Hakuno, Fracture analyses of concrete structures by the modified distinct element method, Structural Engineering/Earthquake Engineering 6 (2) (1989) 283-294.

[34] M. Vassaux, F. Ragueneau, B. Richard, A. Millard, Compressive behavior of a lattice discrete element model for quasi-brittle materials, Computational Modelling of Concrete Structures 1 (2014) 335-344.

[35] M. Vassaux, C. Oliver-Leblond, B. Richard, F. Ragueneau, Beam-particle approach to model cracking and energy dissipation in concrete: Identification strategy and validation, Cement and Concrete Compositions. 
[36] B. Karihaloo, P. Shao, Q. Xiao, Lattice modelling of the failure of particle composites, Engineering Fracture Mechanics 70 (17) (2003) 2385-2406.

[37] D. Grégoire, L. B. Rojas-Solano, G. Pijaudier-Cabot, Failure and size effect for notched and unnotched concrete beams, International Journal for Numerical and Analytical Methods in Geomechanics 37 (10) (2013) 14341452.

[38] M. Briffaut, F. Benboudjema, C. La Borderie, J.-M. Torrenti, Creep consideration effect on meso-scale modeling of concrete hydration process and consequences on the mechanical behavior, Journal of Engineering Mechanics 139 (12) (2013) 1808-1817.

[39] J. Mazars, Application de la mécanique de l'endommagement au comportement non linéaire et à la rupture du béton de structure, Ph.D. thesis, Université Paris VI (1984).

[40] P. H. Feenstra, R. De Borst, A composite plasticity model for concrete, International Journal of Solids and Structures 33 (5) (1996) 707-730.

[41] M. Matallah, C. La Borderie, O. Maurel, A practical method to estimate crack openings in concrete structures, International Journal for Numerical and Analytical Methods in Geomechanics 34 (15) (2010) 1615-1633.

[42] A. Signorini, Questioni di elasticità non linearizzata e semilinearizzata., Rend. Mat. Appl., V. Ser. 18 (1959) 95-139.

[43] P. Rivillon, A. Gabs, Projet national de recherche ceos.fr, axe 2 expérimentations, rapport nºem 09 26023877-c, Tech. rep., Technical Report, CSTB (2011).

[44] Code de calcul aux éléments finis - cast3m, http://www-cast3m.cea.fr/ (2014).

[45] F. C. Filippou, A. Issa, Nonlinear analysis of reinforced concrete frames under cyclic load reversals, Vol. 88, Earthquake Engineering Research Center, University of California, 1988.

[46] B. Richard, F. Ragueneau, C. Cremona, L. Adelaide, J.-L. Tailhan, A three-dimensional steel/concrete interface model including corrosion effects, Engineering Fracture Mechanics 77 (6) (2010) 951-973.

[47] A. Casanova, L. Jason, L. Davenne, Bond slip model for the simulation of reinforced concrete structures, Engineering Structures 39 (2012) 66-78. 